\title{
Atrichia with papular lesions
}

INSERM

\section{Source}

INSERM. (1999). Orphanet: an online rare disease and orphan drug data base. Atrichia with papular lesions. ORPHA:86819

Atrichia with papular lesions is a rare inherited form of alopecia characterized by irreversible hair loss during the neonatal period on all hear-bearing areas of the body, later associated with the development of papular lesions all over the body and preferentially on the face and extensor surfaces of the extremities. 